\title{
Synthesis, Crystal Structure and Fungicidal Activities of New Type Oxazolidinone-Based Strobilurin Analogues
}

\author{
Yuhao Li, Rui Liu, Zhangwei Yan, Xiangning Zhang, ${ }^{\dagger}$ and Hongjun Zhu* \\ Department of Applied Chemistry, College of Science, Nanjing University of Technology, Nanjing 210009, P. R. China \\ ${ }_{\dagger}^{\dagger}$ Jiangsu Pesticide Research Institute Co. Ltd., Nanjing 210019, P. R. China. ${ }^{*}$ E-mail: zhuhjnjut@hotmail.com \\ Received June 7, 2010, Accepted September 15, 2010
}

\begin{abstract}
A series of oxazolidinone-based strobilurin analogues were efficiently synthesized by the reaction of 3-(2-bromomethylphenyl)oxazolidin-2-one with 1-substituted phenyl-2H-pyrazolin-3-one. Their structures were confirmed and characterized by ${ }^{1} \mathrm{H}-\mathrm{NMR},{ }^{13} \mathrm{C}-\mathrm{NMR}$, elemental analysis, and mass spectroscopy. In addition, the crystal structure of the target compound 3-(2-((1-phenyl-2H-pyrazol-3-yloxy)methyl)phenyl) oxazolidin-2-one was determined by single crystal $\mathrm{X}$-ray diffraction. The bioassay results of these compounds indicated that some of the oxazolidin-2-one derivatives containing $\mathrm{N}$-substituted phenyl $2 \mathrm{H}$-pyrazol ring exhibited potential in vivo fungicidal activities against $M$. grisea at the dosage of $1 \mathrm{~g} \mathrm{~L}^{-1}$.
\end{abstract}

Key Words: Strobilurin fungicide, Oxazolidinone, Synthesis, X-ray diffraction, Structure-activity relationships

\section{Introduction}

Strobilurin fungicides are nowadays among the most important fungicides in the market of active agrochemicals. ${ }^{1-4}$ Since 1996 the first strobilurin product commercialized, a mass of synthetic derivatives of the strobilurin family have been prepared and investigated. ${ }^{5-9}$ Pyraclostrobin (Fig. 1), an excellent representative of methoxycarbamates in this family, has shown a broader antifungal activity spectrum, higher efficiency and security than previous fungicides. ${ }^{10,11}$ However, with a large number of strobilurin fungicides being used, a mass increase of plant pathogens against strobilurin fungicides have occurred.

The strobilurin fungicide inhibits mitochondrial respiration which all have a common mode of action by binding to the Qo site of the cytochrome bc1 complex. ${ }^{12}$ Based on modeling of the co-crystal structure with the Qo inhibitor azoxystrobin, binding pocket with amino acids of the cytochrome bc1 enzyme

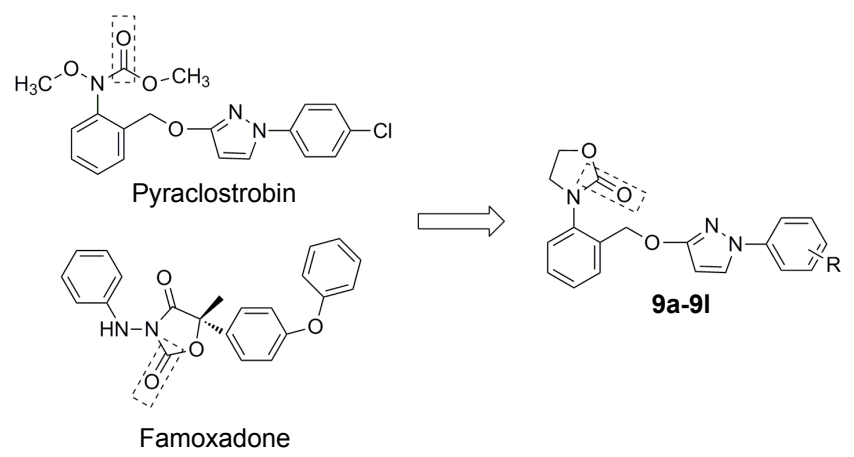

$$
\begin{array}{ll}
\text { 9a: } R=\text { none } & \text { 9g: } R=2-C l \\
\text { 9b: } R=4-C l & 9 h: R=2-B r \\
\text { 9c: } R=3-C l & 9 i: R=4-\mathrm{OCF}_{3} \\
\text { 9d: } R=4-B r & 9 j: R=2-\mathrm{CH}_{3} \\
\text { 9e: } R=3-F & \text { 9k: } R=3-C_{3}-4-F \\
\text { 9f: } R=2-\mathrm{CH}_{2} \mathrm{CH}_{3} & \text { 9l: } \mathrm{R}=3-\mathrm{CH}_{3}
\end{array}
$$

Figure 1. Design strategy of the target compounds (9a-9l). complex showed the carbonyl oxygen moiety of the toxophore may bind with a hydrogen bond to the amide group of glutamine. ${ }^{13}$ Famoxadone (Figure 1), an excellent representative of oxazolidinediones in strobilurin family, ${ }^{13}$ plays a significant role. The bioisosteric relationship identification for the carbonyl group in oxazolidinone ring would seem to be the carbonyl in Famoxadone, and linezolid as the representative of oxazolidinones antibacterial drug approved into market in 2000. So oxazolidinone ring has more potential use in improving biological activities. ${ }^{14-17}$

To solve the resistance problem, and find new fungicides with low toxicities and broad ranges of fungicidal activities, the effective parts of pyraclostrobin and famoxadone were combined, and a series of new type oxazolidin-2-one derivatives containing $\mathrm{N}$-substituted phenyl $2 \mathrm{H}$-pyrazol ring were designed and synthesized, by introducing oxazolidin-2-one ring into core structure and expecting to obtain good antibacterial activities compounds. The single crystal structure of oxazolidin-2-one 9a was proven, not only giving better understanding about the nature of binding of these compounds, but also helping to explore new structure to enhance the fungicidal activities. Meanwhile their antibacterial activities have been investigated with the aim of understanding the structure-fungicidal activity relationships and developing novel fungicides.

\section{Experimental Section}

Materials. Acetone was distilled and dried over $4 \AA$ molecular sieve. Other solvents and reagents were obtained from commercial sources and used without further purification. $G i$ bberella zeae, Rhizoctonia cerealis, Sclerotinia sclerotioru, Botrytis cinerea and Magnaporthe grisea were obtained from Jiangsu Pesticide Research Institute Co. Ltd.

Reactions were monitored by thin-layer chromatography (TLC). Analytical thin-layer chromatography was performed on silica gel GF254. Silica gel (100 - 200 mesh) was used for flash column chromatography. The melting points were measur- 
ed on an X-4 microscope electrothermal apparatus (Taike China) and were uncorrected. The ${ }^{1} \mathrm{H}-\mathrm{NMR}$ spectra and ${ }^{13} \mathrm{C}-\mathrm{NMR}$ spectra were recorded in $\mathrm{CDCl}_{3}$ or DMSO- $d_{6}$ solution on a Bruker AV 300 or AV 500 NMR spectrophotometer with TMS as the internal standard. Elemental analysis were performed on a Vario EL III elemental analysis instrument and the results were within $0.3 \%$ of the calculated value. Mass spectra were recorded with an Agilent 1100 Series LC/MSD Trap SL. X-ray intensity data were recorded on a Nonius CAD4 single crystal diffraction.

\section{General Synthetic Procedure for 4a-4l.}

Substituted phenyl hydrazine hydrochloride (2a-2l): ${ }^{18} \mathrm{~A}$ solution of $\mathrm{NaNO}_{2}(4.77 \mathrm{~g}, 0.069 \mathrm{~mol})$ in water $(20 \mathrm{~mL})$ was added to an ice-cold mixture of substituted aniline in $6 \mathrm{~N} \mathrm{HCl}(100 \mathrm{~mL})$ and stirred for $20 \mathrm{~min}$. The resulted solution was added slowly to a solution of $\mathrm{SnCl}_{2} \cdot 2 \mathrm{H}_{2} \mathrm{O}$ in $37 \% \mathrm{HCl}$ solution $(250 \mathrm{~mL})$ at $-10{ }^{\circ} \mathrm{C}$. The reaction mixture was stirred at $-5{ }^{\circ} \mathrm{C}$ for $2 \mathrm{~h}$. The crude product was collected by filtration, dissolved in methanol, dried over $\mathrm{Na}_{2} \mathrm{SO}_{4}$, filtered, and concentrated. The resulting white solid was washed with diethyl ether and dried under vacuum to obtain compounds. Substituted phenyl hydrazine hydrochlorides were directly used for next step.

1-Substituted pyrazolidin-3-one (3a-3l): ${ }^{19} 0.45 \mathrm{~mol}$ of ethyl acrylate are added dropwise at $40{ }^{\circ} \mathrm{C}-45^{\circ} \mathrm{C}$ for $1 \mathrm{~h}$ to a mixture of $0.2 \mathrm{~mol}$ of sodium methoxide ( $98 \%$ ), $50 \mathrm{~mL}$ of ethanol, $55 \mathrm{~mL}$ of toluene and $0.09 \mathrm{~mol}$ of substituted phenyl hydrazine hydrochloride (2a-2l), and the mixture is subsequently stirred for $15 \mathrm{~h}$ at $45^{\circ} \mathrm{C}$. The reaction mixture is evaporated to $40 \mathrm{~mL}$ and the residue is taken up in sufficient water. The resulting mixture was washed a number of times with toluene and the combined organic phases were extracted with $5 \% \mathrm{NaOH}$ solution. The combined aqueous phases were adjusted to a $\mathrm{pH}$ of 6.5 and cooled to $5{ }^{\circ} \mathrm{C}$. The solid formed was filtered off with suction, washed with water and dried under reduced pressure.

1-Substituted phenyl-2H-pyrazolin-3-one (4a-4l): ${ }^{19} 150 \mathrm{mmol}$ of 1-substituted pyrazolidin-3-one (3a-3l) were dissolved in $100 \mathrm{~mL}$ of dimethylformamide and admixed with $2.4 \mathrm{~g}$ (0.1 equiv, $15 \mathrm{mmol}$ ) of $\mathrm{FeCl}_{3} \cdot 6 \mathrm{H}_{2} \mathrm{O}$. While passing in air, the mixture was heated to $80^{\circ} \mathrm{C}$, this temperature was held for $4 \mathrm{~h}$ and the mixture was stirred for further $12 \mathrm{~h}$ without heating. The reaction mixture was poured into water and stirred for $2 \mathrm{~h}$, the precipitate formed was filtered off, washed with water and dried under reduced pressure to got product.

1-Phenyl-2H-pyrazolin-3-one (4a): Gray solid, mp 155 $156{ }^{\circ} \mathrm{C}$ (decomposition), ${ }^{1} \mathrm{H}-\mathrm{NMR}$ (DMSO- $\left.d_{6}\right) \delta 5.80(\mathrm{~d}, J=$ $1.6 \mathrm{~Hz}, 1 \mathrm{H}, \mathrm{CH}), 7.17$ (t, $J=7.2 \mathrm{~Hz}, 1 \mathrm{H}, \mathrm{Ar}-\mathrm{H}), 7.42$ (t, $J=7.6$ $\mathrm{Hz}, 1 \mathrm{H}, \mathrm{Ar}-\mathrm{H}), 7.67$ (d, $J=8.1 \mathrm{~Hz}, 1 \mathrm{H}, \mathrm{Ar}-\mathrm{H}), 8.21$ (d, $J=1.6$

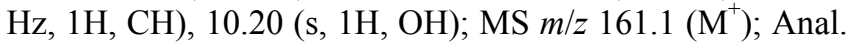
calcd. for $\mathrm{C}_{9} \mathrm{H}_{8} \mathrm{~N}_{2} \mathrm{O}: \mathrm{C} 67.49, \mathrm{H} 5.03, \mathrm{~N} 17.49$, found C 67.47, H 5.05, N 17.48.

1-(4-Chlorophenyl)-2H-pyrazolin-3-one (4b): Gray solid, $\mathrm{mp}$ $189-191{ }^{\circ} \mathrm{C}$ (decomposition), ${ }^{1} \mathrm{H}-\mathrm{NMR}\left(\mathrm{CDCl}_{3}\right) \delta 5.92(\mathrm{~d}, J=$ $2.1 \mathrm{~Hz}, 1 \mathrm{H}, \mathrm{CH}), 7.44$ (s, 4H, Ar-H), 7.64 (d, J=2.1 Hz, 1H, $\mathrm{CH}), 11.47$ (s, 1H, OH); MS m/z $195.0\left(\mathrm{M}^{+}\right)$; Anal. calcd for $\mathrm{C}_{9} \mathrm{H}_{7} \mathrm{ClN}_{2} \mathrm{O}: \mathrm{C} 55.54, \mathrm{H} 3.63, \mathrm{~N} 14.39$, found C 55.52, H 3.66, $\mathrm{N} 14.38$.

1-(3-Chlorophenyl)-2H-pyrazolin-3-one (4c): Gray solid, mp $132-135{ }^{\circ} \mathrm{C}$ (decomposition), ${ }^{1} \mathrm{H}-\mathrm{NMR}$ (DMSO- $\left.d_{6}\right) \delta 5.85$ (d,
$J=2.2 \mathrm{~Hz}, 1 \mathrm{H}, \mathrm{CH}), 7.22(\mathrm{t}, J=7.7 \mathrm{~Hz}, 1 \mathrm{H}, \mathrm{Ar}-\mathrm{H}), 7.45(\mathrm{t}, J=$ $8.1 \mathrm{~Hz}, 1 \mathrm{H}, \mathrm{Ar}-\mathrm{H}), 7.66$ (d, $J=8.1 \mathrm{~Hz}, 1 \mathrm{H}, \mathrm{Ar}-\mathrm{H}), 7.78$ (s, 1H, Ar-H), 8.30 (d, $J=2.2 \mathrm{~Hz}, 1 \mathrm{H}, \mathrm{CH}), 10.35$ (s, 1H, OH); MS $m / z 195.0\left(\mathrm{M}^{+}\right)$; Anal. calcd for $\mathrm{C}_{9} \mathrm{H}_{7} \mathrm{ClN}_{2} \mathrm{O}$ : C 55.54, H 3.63, $\mathrm{N}$ 14.39, found C 55.53, H 3.65, N 14.36.

1-(4-Bromophenyl)-2H-pyrazolin-3-one (4d): Gray solid, $\mathrm{mp}$ $221-223{ }^{\circ} \mathrm{C}$ (decomposition), ${ }^{1} \mathrm{H}-\mathrm{NMR}$ (DMSO- $\left.d_{6}\right) \delta 5.83(\mathrm{~d}$, $J=2.4 \mathrm{~Hz}, 1 \mathrm{H}, \mathrm{CH}), 7.59-7.65$ (m, 4H, Ar-H), 8.23 (d, $J=2.4$ $\mathrm{Hz}, 1 \mathrm{H}, \mathrm{CH}), 10.28$ (s, 1H, OH), MS m/z $238.9\left(\mathrm{M}^{+}\right)$; Anal. calcd for $\mathrm{C}_{9} \mathrm{H}_{7} \mathrm{BrN}_{2} \mathrm{O}: \mathrm{C} 45.22, \mathrm{H} 2.95, \mathrm{~N} 11.72$, found $\mathrm{C} 45.20, \mathrm{H}$ 2.98, N 11.71 .

1-(3-Fluorophenyl)-2H-pyrazolin-3-one (4e): Gray solid, mp $123-125^{\circ} \mathrm{C}$ (decomposition), ${ }^{1} \mathrm{H}-\mathrm{NMR}$ (DMSO- $\left.d_{6}\right) \delta 5.85$ (d, $J=2.2 \mathrm{~Hz}, 1 \mathrm{H}, \mathrm{CH}), 7.00$ (S, 1H, Ar-H), 7.49 (d, 3H, Ar-H), $8.28(\mathrm{~d}, J=2.2,1 \mathrm{H}, \mathrm{CH}), 10.35(\mathrm{~s}, 1 \mathrm{H}, \mathrm{OH}) ; \mathrm{MS} m / z 179.1\left(\mathrm{M}^{+}\right)$; Anal. calcd for $\mathrm{C}_{9} \mathrm{H}_{7} \mathrm{FN}_{2} \mathrm{O}$ : C 60.67, H 3.96, N 8.98, found $\mathrm{C}$ 60.68 H 3.99 N 8.97.

1-(2-Ethylphenyl)-2H-pyrazolin-3-one (4f): Gray solid, mp $142-144{ }^{\circ} \mathrm{C}$ (decomposition), ${ }^{1} \mathrm{H}-\mathrm{NMR}$ (DMSO- $\left.d_{6}\right) \delta 1.03(\mathrm{t}$, $\left.J=7.5 \mathrm{~Hz}, 3 \mathrm{H}, \mathrm{CH}_{3}\right), 2.62\left(\mathrm{q}, J=7.5 \mathrm{~Hz}, 2 \mathrm{H}, \mathrm{CH}_{2}\right), 5.71(\mathrm{~d}$, $J=2.4 \mathrm{~Hz}, 1 \mathrm{H}, \mathrm{CH}), 7.24$ (dd, $J=7.7 \mathrm{~Hz}, 1 \mathrm{H}, \mathrm{Ar}-\mathrm{H}), 7.28(\mathrm{td}$, $J=7.3 \mathrm{~Hz}, 1 \mathrm{H}, \mathrm{Ar}-\mathrm{H}), 7.32(\mathrm{td}, J=7.3 \mathrm{~Hz}, 1 \mathrm{H}, \mathrm{Ar}-\mathrm{H}), 7.36$ (dd, $J=7.7 \mathrm{~Hz}, 1 \mathrm{H}, \mathrm{Ar}-\mathrm{H}), 7.63$ (d, $J=2.4 \mathrm{~Hz}, 1 \mathrm{H}, \mathrm{CH}), 9.86$ (s, $1 \mathrm{H}, \mathrm{OH})$; MS $m / z 189.1\left(\mathrm{M}^{+}\right)$; Anal. calcd for $\mathrm{C}_{11} \mathrm{H}_{12} \mathrm{~N}_{2} \mathrm{O}$ : C 70.19, H 6.43, N 14.88, found C 70.19, H 6.44, N 14.87.

1-(2-Chlorophenyl)-2H-pyrazolin-3-one (4g): Gray solid, mp 163 - $168{ }^{\circ} \mathrm{C}$ (decomposition), ${ }^{1} \mathrm{H}-\mathrm{NMR}$ (DMSO- $\left.d_{6}\right) \delta 5.79(\mathrm{~d}$, $J=2.4 \mathrm{~Hz}, 1 \mathrm{H}, \mathrm{CH}), 7.37$ (t, $J=7.2 \mathrm{~Hz}, 1 \mathrm{H}, \mathrm{Ar}-\mathrm{H}), 7.45$ (t, $J=$ $7.2 \mathrm{~Hz}, 1 \mathrm{H}, \mathrm{Ar}-\mathrm{H}), 7.52$ (d, $J=7.1 \mathrm{~Hz}, 1 \mathrm{H}, \mathrm{Ar}-\mathrm{H}), 7.60$ (d, $J=$ $7.1 \mathrm{~Hz}, 1 \mathrm{H}, \mathrm{Ar}-\mathrm{H}), 7.85$ (d, $J=2.4 \mathrm{~Hz}, 1 \mathrm{H}, \mathrm{CH}), 10.13(\mathrm{~s}, 1 \mathrm{H}$, $\mathrm{OH})$; $\mathrm{MS} m / z$ 195.0 $\left(\mathrm{M}^{+}\right)$; Anal. calcd for $\mathrm{C}_{9} \mathrm{H}_{7} \mathrm{ClN}_{2} \mathrm{O}$ : C 55.54, H 3.63, N 14.39, found C 55.52, H 3.68, N 14,37.

1-(2-Bromophenyl)-2H-pyrazolin-3-one (4h): Gray solid, mp $174-177^{\circ} \mathrm{C}$ (decomposition), ${ }^{1} \mathrm{H}-\mathrm{NMR}$ (DMSO- $\left.d_{6}\right) \delta 5.78$ (d, $J=2.4 \mathrm{~Hz}, 1 \mathrm{H}, \mathrm{CH}), 7.36(\mathrm{t}, J=7.2 \mathrm{~Hz}, 1 \mathrm{H}, \mathrm{Ar}-\mathrm{H}), 7.43(\mathrm{t}, J=$ $7.2 \mathrm{~Hz}, 1 \mathrm{H}, \mathrm{Ar}-\mathrm{H}), 7.53$ (d, $J=7.1 \mathrm{~Hz}, 1 \mathrm{H}, \mathrm{Ar}-\mathrm{H}), 7.63(\mathrm{~d}, J=$ $7.1 \mathrm{~Hz}, 1 \mathrm{H}, \mathrm{Ar}-\mathrm{H}), 7.87$ (d, $J=2.4 \mathrm{~Hz}, 1 \mathrm{H}, \mathrm{CH}), 10.10$ (s, 1H, $\mathrm{OH})$; $\mathrm{MS} m / z 238.9\left(\mathrm{M}^{+}\right)$; Anal. calcd for $\mathrm{C}_{9} \mathrm{H}_{7} \mathrm{BrN}_{2} \mathrm{O}$ : C 45.22, $\mathrm{H} 2.95, \mathrm{~N} 11.72$, found C 45.22, H 2.97, N 11.71.

1-(4-(Trifluoromethoxy)phenyl)-2H-pyrazolin-3-one (4i): Gray solid, mp $142-144{ }^{\circ} \mathrm{C}$ (decomposition), ${ }^{1} \mathrm{H}-\mathrm{NMR}$ (DMSO$\left.d_{6}\right) \delta 5.84(\mathrm{~d}, J=2.3 \mathrm{~Hz}, 1 \mathrm{H}, \mathrm{CH}), 7.37(\mathrm{~d}, J=8.6 \mathrm{~Hz}, 2 \mathrm{H}, \mathrm{Ar}-$ H), 7.78 (d, $J=9.1 \mathrm{~Hz}, 2 \mathrm{H}, \operatorname{Ar}-\mathrm{H}), 8.25$ (d, $J=2.4 \mathrm{~Hz}, 1 \mathrm{H}$, $\mathrm{CH}), 10.32$ (s, 1H, OH); MS $m / z 245.0\left(\mathrm{M}^{+}\right)$; Anal. calcd for $\mathrm{C}_{10} \mathrm{H}_{7} \mathrm{~F}_{3} \mathrm{~N}_{2} \mathrm{O}_{2}$ : C 49.19, H 2.89, N 11.47, found C 49.20, H 2.92, $\mathrm{N} 11.45$.

1-(2-Methylphenyl)-2H-pyrazolin-3-one (4j): Gray solid, mp $128-131{ }^{\circ} \mathrm{C}$ (decomposition), ${ }^{1} \mathrm{H}-\mathrm{NMR}$ (DMSO- $d_{6}$ ) $\delta 2.27$ (s, $\left.3 \mathrm{H}, \mathrm{CH}_{3}\right), 5.73(\mathrm{~d}, J=1.6 \mathrm{~Hz}, 1 \mathrm{H}, \mathrm{CH}), 7.25-7.33$ (m, 4H, ArH), 7.69 (d, $J=1.7 \mathrm{~Hz}, 1 \mathrm{H}, \mathrm{CH}), 9.93(\mathrm{~s}, 1 \mathrm{H}, \mathrm{OH}) ; \mathrm{MS} m / z 175.1$ $\left(\mathrm{M}^{+}\right)$; Anal. calcd for $\mathrm{C}_{10} \mathrm{H}_{10} \mathrm{~N}_{2} \mathrm{O}$ : C 68.95, H 5.79, N 16.08, found C 68.96, H 5.80, N 16.06.

1-(4-Fluoro-3-trifluoromethylphenyl)-2H-pyrazolin-3-one (4k): Gray solid, mp $129-133{ }^{\circ} \mathrm{C}$ (decomposition); ${ }^{1} \mathrm{H}-\mathrm{NMR}$ $\left(\mathrm{DMSO}-d_{6}\right) \delta 5.90(\mathrm{~d}, J=2.2 \mathrm{~Hz}, 1 \mathrm{H}, \mathrm{CH}), 7.77(\mathrm{~d}, J=8.5 \mathrm{~Hz}$, $1 \mathrm{H}, \mathrm{Ar}-\mathrm{H}), 7.99$ (t, $J=8.8 \mathrm{~Hz}, 1 \mathrm{H}, \mathrm{Ar}-\mathrm{H}), 8.09$ (s, $1 \mathrm{H}, \mathrm{Ar}-\mathrm{H})$, 8.40 (d, $J=2.1 \mathrm{~Hz}, 1 \mathrm{H}, \mathrm{CH}), 10.48$ (s, 1H, OH); MS $m / z 247.0$ $\left(\mathrm{M}^{+}\right)$; Anal. Calcd for $\mathrm{C}_{10} \mathrm{H}_{6} \mathrm{~F}_{4} \mathrm{~N}_{2} \mathrm{O}: \mathrm{C} 48.59, \mathrm{H} 2.85, \mathrm{~N} 11.33$, 
found C 48.57, H 2.88, N 11.33.

1-(3-Methylphenyl)-2H-pyrazolin-3-one (4I): Gray solid, mp $112-114{ }^{\circ} \mathrm{C}$ (decomposition), ${ }^{1} \mathrm{H}-\mathrm{NMR}$ (DMSO- $\left.d_{6}\right) \delta 2.35$ (s, $\left.3 \mathrm{H}, \mathrm{CH}_{3}\right), 5.79(\mathrm{~d}, J=2.2 \mathrm{~Hz}, 1 \mathrm{H}, \mathrm{CH}), 7.00(\mathrm{~s}, 1 \mathrm{H}, \mathrm{Ar}-\mathrm{H})$, 7.29 (s, 1H, Ar-H), 7.48 (d, 2H, Ar-H), 8.18 (d, J=2.2 Hz, 1H, $\mathrm{CH}), 10.17$ (s, 1H, OH); MS m/z $175.1\left(\mathrm{M}^{+}\right)$; Anal. calcd for $\mathrm{C}_{10} \mathrm{H}_{10} \mathrm{~N}_{2} \mathrm{O}$ : C 68.95, H 5.79, N 16.08, found C 68.92, H 5.83, $\mathrm{N} 16.07$.

2-Chloroethyl-o-tolylcarbamate (6): ${ }^{20}$ A three neck, $250 \mathrm{~mL}$ round bottom flask fitted with a mechanical stirrer, dropping funnel, and thermometer was charged with 2-methylaniline $(10.7 \mathrm{~g}, 0.1 \mathrm{~mol})$, dichloromethane $(150 \mathrm{~mL})$ and $10 \%$ aqueous $\mathrm{NaOH}$ solution $(44 \mathrm{~mL}, 1.1 \mathrm{~mol})$. 2-chloroethyl chloroformate $(10.8 \mathrm{~mL}, 0.11 \mathrm{~mol})$ was added dropwise to the vigorously stirred reaction mixture over 20 min maintaining an internal temperature of $25{ }^{\circ} \mathrm{C}$. At the end of the addition, the $\mathrm{pH}$ of the aqueous layer was 8 . After stirring an additional $15 \mathrm{~min}$, the $\mathrm{pH}$ of the aqueous layer (now 5 - 6) was adjusted to 11 with $5 \mathrm{~mL}$ of $10 \%$ aqueous $\mathrm{NaOH}$ solution, and the mixture was stirred an additional $4 \mathrm{~h}$ at refluxing temperature, monitored by TLC. After cooling to room temperature, the organic layer washed with water $(200 \mathrm{~mL} \times 3)$, dried over $\mathrm{MgSO}_{4}$, filtered and evaporated to afford residue. Yield $98 \%$, white solid, mp $41-42{ }^{\circ} \mathrm{C}$, ${ }^{1} \mathrm{H}-\mathrm{NMR}\left(\mathrm{CDCl}_{3}\right) \delta 2.26\left(\mathrm{~s}, 3 \mathrm{H}, \mathrm{CH}_{3}\right), 3.73(\mathrm{t}, J=5.7 \mathrm{~Hz}, 2 \mathrm{H}$, $\left.\mathrm{CH}_{2}\right), 4.42\left(\mathrm{t}, J=5.7 \mathrm{~Hz}, 2 \mathrm{H}, \mathrm{CH}_{2}\right), 6.47-7.23(\mathrm{~m}, 4 \mathrm{H}, \mathrm{Ar}-\mathrm{H})$, $7.74(\mathrm{~s}, 1 \mathrm{H}, \mathrm{NH})$; $\mathrm{MS} m / z 214.1\left(\mathrm{M}^{+}\right)$; Anal. calcd for $\mathrm{C}_{10} \mathrm{H}_{12^{-}}$ $\mathrm{ClNO}_{2}$ : C 55.95, H 6.10, N 6.52, found C 55.91, H 6.14, N 16.50.

3-(o-Tolyl)oxazolidin-2-one (7): ${ }^{21}$ A $250 \mathrm{~mL}, 3$ neck round bottom flask fitted with a thermometer, mechanical stirrer, and dropping funnel was charged with $78.1 \mathrm{~g}(0.344 \mathrm{~mol})$ of compound 6 and $200 \mathrm{~mL}$ of methanol. The white solution was treated with $82.5 \mathrm{~mL}$ of $25 \%$ methanolic $\mathrm{NaOCH}_{3}$, over 5 min maintaining an internal temperature less than $30{ }^{\circ} \mathrm{C}$. After $5 \mathrm{~h}$ the reaction mixture was poured into water and extracted with $\mathrm{CH}_{2} \mathrm{Cl}_{2}(3 \times 300 \mathrm{~mL})$. The combined organic extracts were dried over $\mathrm{MgSO}_{4}$, filtered and evaporated to afford $62.8 \mathrm{~g}$ of 7 as a colorless oil. Yield 95.7\%, ${ }^{1} \mathrm{H}-\mathrm{NMR}\left(\mathrm{CDCl}_{3}\right) \delta 2.30(\mathrm{~s}$, $\left.3 \mathrm{H}, \mathrm{CH}_{3}\right), 3.92\left(\mathrm{t}, J=7.6 \mathrm{~Hz}, 2 \mathrm{H}, \mathrm{CH}_{2}\right), 4.49(\mathrm{t}, J=7.6 \mathrm{~Hz}, 2 \mathrm{H}$, $\left.\mathrm{CH}_{2}\right)$, 7.22-7.27 (m, 4H, Ar-H); MS m/z 178.1 ( $\left.\mathrm{M}^{+}\right)$; Anal. calcd for $\mathrm{C}_{10} \mathrm{H}_{11} \mathrm{NO}_{2}$ : C 67.40, H 6.79, N 7.86, found C 67.40, H 6.80, $\mathrm{N} 7.85$.

3-(2-(Bromomethyl)phenyl)oxazolidin-2-one (8): ${ }^{22}$ To the solution of compound $7(10.46 \mathrm{~g}, 59.0 \mathrm{mmol})$ in $\mathrm{CCl}_{4}(150 \mathrm{~mL})$ was added $N$-bromosuccinimide ( 1 equiv., $10.45 \mathrm{~g}, 59.0 \mathrm{mmol}$ ) and azobisisobutyronitrile $(0.1$ equiv, $0.97 \mathrm{~g}, 5.9 \mathrm{mmol})$. The reaction mixture was stirred and refluxed for $1 \mathrm{~h}$ without light then filtered to remove succinimide and washed with brine $(100 \mathrm{~mL} \times 3)$. The organic layer was dried over $\mathrm{MgSO}_{4}$, filtered and evaporated to afford residue. The residue was purified by silica gel column chromatography (eluent: petroleum ether/ethyl acetate $=2: 1, \mathrm{v} / \mathrm{v}$ ) to afford $9.1 \mathrm{~g}$ of the title compound as a white solid. Yield, $60 \%$, mp $79-84{ }^{\circ} \mathrm{C},{ }^{1} \mathrm{H}-\mathrm{NMR}\left(\mathrm{CDCl}_{3}\right) \delta 4.10$ $\left(\mathrm{t}, J=7.9 \mathrm{~Hz}, 2 \mathrm{H}, \mathrm{CH}_{2}\right), 4.58\left(\mathrm{t}, J=7.9 \mathrm{~Hz}, 2 \mathrm{H}, \mathrm{CH}_{2}\right), 4.58$ (s, $\left.2 \mathrm{H}, \mathrm{Ar}-\mathrm{CH}_{2} \mathrm{Br}\right), 7.23-7.47$ (m, $\left.4 \mathrm{H}, \mathrm{Ar}-\mathrm{H}\right) ;{ }^{13} \mathrm{C}-\mathrm{NMR}\left(\mathrm{CDCl}_{3}\right)$ $\delta 30.01,46.92,63.17,117.96,129.78,129.81,131.93,136.04$, 136.26. MS $m / z$ 256.0(M $\left.\mathrm{M}^{+}\right), 146.1,107.1$; Anal. calcd for $\mathrm{C}_{10} \mathrm{H}_{10}$ $\mathrm{BrNO}_{2}$ : C 46.90, H 3.94, N 5.47, found C 46.87, H 3.96, N 5.43.

3-(2-((1-Substituted phenyl-2H-pyrazol-3-yloxy)methyl) phenyl) oxazolidin-2-one (9a-9l): ${ }^{23}$ A mixture of compound 4 ( $3.0 \mathrm{mmol}$ ) and anhydrous $\mathrm{K}_{2} \mathrm{CO}_{3}$ (1.5 equiv., $0.63 \mathrm{~g}, 4.5 \mathrm{mmol}$ ) in dry acetone $(20 \mathrm{~mL})$ was stirred and refluxed for $1 \mathrm{~h}$. Then, the solution $0.77 \mathrm{~g}$ (1.0 equiv, $3.0 \mathrm{mmol})$ of $\mathbf{8}$ in dry acetone $(20 \mathrm{~mL})$ was added. The mixture was reacted for $12 \mathrm{~h}$ at the refluxing temperature. The resulting mixture was cooled to room temperature and filtered. The filtrate was added chloroform $(50 \mathrm{~mL})$ and washed with water $(2 \times 50 \mathrm{~mL})$. The organic layer was dried over $\mathrm{MgSO}_{4}$, filtered and evaporated to afford residue which was purified by silica gel column chromatography (eluent: petroleum ether/ethyl acetate $=2: 1, \mathrm{v} / \mathrm{v}$ ) monitoring by TLC. The pure fractions were combined and evaporated to afford the target compounds. The following compounds 9a-9l were prepared according to this procedure.

3-(2-((1-Phenyl-2H-pyrazol-3-yloxy)methyl)phenyl)oxazolidin-2-one (9a): A white solid, yield $88.5 \%, \mathrm{mp} 105-106^{\circ} \mathrm{C}$, ${ }^{1} \mathrm{H}-\mathrm{NMR}\left(\mathrm{CDCl}_{3}\right) \delta 4.00\left(\mathrm{t}, J=7.8,2 \mathrm{H}, \mathrm{CH}_{2}\right), 4,48(\mathrm{t}, J=7.8$, $\left.2 \mathrm{H}, \mathrm{CH}_{2}\right), 5.37\left(\mathrm{~s}, 2 \mathrm{H}, \mathrm{CH}_{2}\right), 5.92(\mathrm{~d}, J=2.6,1 \mathrm{H}, \mathrm{CH}), 7.19-$ $7.66(\mathrm{~m}, 9 \mathrm{H}, \mathrm{Ar}-\mathrm{H}), 7.75(\mathrm{~d}, J=2.6,1 \mathrm{H}, \mathrm{CH}) ;{ }^{13} \mathrm{C}-\mathrm{NMR}\left(\mathrm{CDCl}_{3}\right)$ $\delta$ 48.77, 62.49, 67.72, 93.79, 117.84, 125.43, 126.81, 127.81, 128.36, 129.34, 129.47, 130.66, 134.98, 136.40, 140.07, 156.99, 164.03; MS $m / z$ 336.1( $\left.\mathrm{M}^{+}\right)$176.0; Anal. calcd for $\mathrm{C}_{19} \mathrm{H}_{17} \mathrm{~N}_{3} \mathrm{O}_{3}$ : C 68.05, H 5.11 N 12.53, found C 68.01, H 5.16, N 12.49.

3-(2-((1-(4-Chlorophenyl)-2H-pyrazol-3-yloxy)methyl) phenyl)oxazolidin-2-one (9b): A white solid, yield $86 \%, \mathrm{mp}$ $138-139{ }^{\circ} \mathrm{C},{ }^{1} \mathrm{H}-\mathrm{NMR}\left(\mathrm{CDCl}_{3}\right) \delta 4.03\left(\mathrm{t}, J=7.8,2 \mathrm{H}, \mathrm{CH}_{2}\right), 4.47$ $\left(\mathrm{t}, J=7.8,2 \mathrm{H}, \mathrm{CH}_{2}\right), 5.35\left(\mathrm{~s}, 2 \mathrm{H}, \mathrm{CH}_{2}\right), 5.93(\mathrm{~d}, J=2.6,1 \mathrm{H}, \mathrm{CH})$, 7.30-7.65 (m, 8H, Ar-H), $7.70(\mathrm{~d}, J=2.6,1 \mathrm{H}, \mathrm{CH}) ;{ }^{13} \mathrm{C}-\mathrm{NMR}$ $\left(\mathrm{CDCl}_{3}\right) \delta 48.83,62.54,67.84,94.38,118.98,125.66,126.83$, $127.85,128.44,129.45,129.58,130.69,134.87,136.40,140.88$, 160.35, 164.2, MS m/z 370.1(M $\left.\mathrm{M}^{+}\right)$, 176.0; Anal. calcd for $\mathrm{C}_{19} \mathrm{H}_{16^{-}}$ $\mathrm{ClN}_{3} \mathrm{O}_{3}$ : C 61.71, $\mathrm{H} 4.36, \mathrm{~N} 11.36$, found $\mathrm{C}, 61.68, \mathrm{H}, 4.40$, $\mathrm{N}, 11.32$.

3-(2-((1-(3-Chlorophenyl)-2H-pyrazol-3-yloxy)methyl) phenyl)oxazolidin-2-one (9c): A white solid, yield 89.0\%, mp $131-132{ }^{\circ} \mathrm{C},{ }^{1} \mathrm{H}-\mathrm{NMR}\left(\mathrm{CDCl}_{3}\right) \delta 4.05\left(\mathrm{t}, J=7.9,2 \mathrm{H}, \mathrm{CH}_{2}\right)$, $4.50\left(\mathrm{t}, J=7.9,2 \mathrm{H}, \mathrm{CH}_{2}\right), 5.36\left(\mathrm{~s}, 2 \mathrm{H}, \mathrm{CH}_{2}\right), 5.93(\mathrm{~d}, J=2.6$, $1 \mathrm{H}, \mathrm{CH}), 7.16-7.65(\mathrm{~m}, 8 \mathrm{H}, \mathrm{Ar}-\mathrm{H}), 7.71(\mathrm{~d}, J=2.6,1 \mathrm{H}, \mathrm{CH})$; ${ }^{13} \mathrm{C}-\mathrm{NMR}\left(\mathrm{CDCl}_{3}\right) \delta 48.80,62.55,67.78,94.72,115.59,118.09$, $125.33,126.73,127.97,128.41,129.54,130.43,130.62,134.84$, 135.22, 136.39, 141.03, 164.22; MS m/z 370.0(M $\left.{ }^{+}\right), 176.0$; Anal. Calcd for $\mathrm{C}_{19} \mathrm{H}_{16} \mathrm{ClN}_{3} \mathrm{O}_{3}$ : C 61.71, H 4.36, $\mathrm{N} 11.36$, found $\mathrm{C}$ 61.68, H 4.40, N 11.31.

3-(2-((1-(4-Bromophenyl)-2H-pyrazol-3-yloxy)methyl) phenyl)oxazolidin-2-one (9d): A white solid, yield $86.0 \%, \mathrm{mp}$ $174-175^{\circ} \mathrm{C},{ }^{1} \mathrm{H}-\mathrm{NMR}\left(\mathrm{CDCl}_{3}\right) \delta 4.03\left(\mathrm{t}, J=7.9,2 \mathrm{H}, \mathrm{CH}_{2}\right), 4.48$ (t, $\left.J=7.9,2 \mathrm{H}, \mathrm{CH}_{2}\right), 5.35\left(\mathrm{~s}, 2 \mathrm{H}, \mathrm{CH}_{2}\right), 5.93(\mathrm{~d}, J=2.7,1 \mathrm{H}$, $\mathrm{CH}), 7.29-7.65(\mathrm{~m}, 8 \mathrm{H}, \mathrm{Ar}-\mathrm{H}), 7.70(\mathrm{~d}, J=2.7,1 \mathrm{H}, \mathrm{CH}) ;{ }^{13} \mathrm{C}-$ NMR $\left(\mathrm{CDCl}_{3}\right) \delta, 48.8362 .54,67.87,94.50,118.45,119.32$, $126.84,127.85,128.44,129.59,130.69,132.42,134.90,136.44$, 139.17, 157.01, 164.27; MS m/z 4.0(M $\left.{ }^{+}\right)$, 176.0; Anal. calcd for $\mathrm{C}_{19} \mathrm{H}_{16} \mathrm{BrN}_{3} \mathrm{O}_{3}$ : C 55.09, H 3.89, N 10.14, found $\mathrm{C} 55.06, \mathrm{H}$ 3.93, N 10.10 .

3-(2-((1-(3-Fluorophenyl)-2H-pyrazol-3-yloxy)methyl) phenyl)oxazolidin-2-one (9e): A white solid, yield $81.0 \%, \mathrm{mp}$ $91-93{ }^{\circ} \mathrm{C},{ }^{1} \mathrm{H}-\mathrm{NMR}\left(\mathrm{CDCl}_{3}\right) \delta 4.00\left(\mathrm{t}, J=7.9,2 \mathrm{H}, \mathrm{CH}_{2}\right), 4.44$ $\left(\mathrm{t}, J=7.9,2 \mathrm{H}, \mathrm{CH}_{2}\right), 5.35\left(\mathrm{~s}, 2 \mathrm{H}, \mathrm{CH}_{2}\right), 5.91(\mathrm{~d}, J=2.6,1 \mathrm{H}, \mathrm{CH})$, 6.85-7.63 (m, 8H, Ar-H), $7.71(\mathrm{~d}, J=2.6,1 \mathrm{H}, \mathrm{CH}) ;{ }^{13} \mathrm{C}-\mathrm{NMR}$ 
$\left(\mathrm{CDCl}_{3}\right) \delta 48.57,62.42,67.59,94.50,105.42,111.69,111.97$, $112.65,126.56,127.95,128.18,129.33,130.33,130.47,130.59$, 134.64, 156.84, 163.99, 164.81; MS m/z 354.0(M $\left.\mathrm{M}^{+}\right)$, 176.0; Anal. calcd for $\mathrm{C}_{19} \mathrm{H}_{16} \mathrm{FN}_{3} \mathrm{O}_{3}: \mathrm{C} 64.58, \mathrm{H} 4.56, \mathrm{~N} 11.89$, found $\mathrm{C} 64.55$, $\mathrm{H} 4.59$, N 11.85 .

3-(2-((1-(2-Ethylphenyl)-2H-pyrazol-3-yloxy)methyl)phenyl)oxazolidin-2-one (9f): A colorless oil, yield 83.9\%, ${ }^{1} \mathrm{H}-\mathrm{NMR}$ $\left(\mathrm{CDCl}_{3}\right) \delta 1.07\left(\mathrm{t}, J=7.6,3 \mathrm{H}, \mathrm{CH}_{3}\right), 2.58\left(\mathrm{q}, J=7.6,2 \mathrm{H}, \mathrm{CH}_{2}\right)$, $3.97\left(\mathrm{t}, J=7.9,2 \mathrm{H}, \mathrm{CH}_{2}\right), 4.41\left(\mathrm{t}, J=7.9,2 \mathrm{H}, \mathrm{CH}_{2}\right), 5.30(\mathrm{~s}, 2 \mathrm{H}$, $\left.\mathrm{CH}_{2}\right), 5.87(\mathrm{~d}, J=2.4,1 \mathrm{H}, \mathrm{CH}), 7.20-7.45(\mathrm{~m}, 8 \mathrm{H}, \mathrm{Ar}-\mathrm{H}), 7.61$ $(\mathrm{d}, J=2.4,1 \mathrm{H}, \mathrm{CH}) ;{ }^{13} \mathrm{C}-\mathrm{NMR}\left(\mathrm{CDCl}_{3}\right) \delta 14.27,24.45,48.53$, $62.38,67.44,91.90,126.04,126.33,126.61,128.13,128.21$, 129.12, 129.65, 130.16, 131.22, 131.96, 135.02, 136.15, 139.80, 156.85, 163.34; $\mathrm{MS} m / z$ 364.1( $\left.\mathrm{M}^{+}\right)$, 176.0; Anal. calcd for. $\mathrm{C}_{21} \mathrm{H}_{21} \mathrm{~N}_{3} \mathrm{O}_{3}$ : C 69.41, H 5.82, N 11.56, found C 69.38, H 5.86, $\mathrm{N} 11.55$.

3-(2-((1-(2-Chlorophenyl)-2H-pyrazol-3-yloxy)methyl) phenyl)oxazolidin-2-one (9g): A white solid, yield 84.0\%, mp $127-128{ }^{\circ} \mathrm{C},{ }^{1} \mathrm{H}-\mathrm{NMR}\left(\mathrm{CDCl}_{3}\right) \delta 3.95\left(\mathrm{t}, J=7.9,2 \mathrm{H}, \mathrm{CH}_{2}\right)$, $4.36\left(\mathrm{t}, J=7.9,2 \mathrm{H}, \mathrm{CH}_{2}\right), 5.27\left(\mathrm{~s}, 2 \mathrm{H}, \mathrm{CH}_{2}\right), 5.86(\mathrm{~d}, J=2.5$, $1 \mathrm{H}, \mathrm{CH}), 7.16-7.58(\mathrm{~m}, 8 \mathrm{H}, \mathrm{Ar}-\mathrm{H}), 7.67(\mathrm{~d}, J=2.5,1 \mathrm{H}, \mathrm{CH})$; ${ }^{13} \mathrm{C}-\mathrm{NMR}\left(\mathrm{CDCl}_{3}\right) \delta 37.79,50.09,63.92,69.00,94.57,127.81$, 128.16, 128.33, 128.34, 129.08, 130.78, 131.82, 132.08, 132.72, $134.40,136.35,137.73,139.33,163.87$; $\mathrm{MS} m / z$ 370.0( $\left.{ }^{+}\right)$, 176.0; Anal. calcd for $\mathrm{C}_{19} \mathrm{H}_{16} \mathrm{ClN}_{3} \mathrm{O}_{3}$ : C 61.71, H 4.36, N 11.36, found $\mathrm{C} 61.69, \mathrm{H} 4.40, \mathrm{~N} 11.33$.

3-(2-((1-(2-Bromophenyl)-2H-pyrazol-3-yloxy)methyl) phenyl)oxazolidin-2-one (9h): A white solid, yield 79.6\%, mp $168-169{ }^{\circ} \mathrm{C},{ }^{1} \mathrm{H}-\mathrm{NMR}\left(\mathrm{CDCl}_{3}\right) \delta 4.00\left(\mathrm{t}, J=7.9,2 \mathrm{H}, \mathrm{CH}_{2}\right)$, $4.43\left(\mathrm{t}, J=7.9,2 \mathrm{H}, \mathrm{CH}_{2}\right), 5.33\left(\mathrm{~s}, 2 \mathrm{H}, \mathrm{CH}_{2}\right), 5.91(\mathrm{~d}, J=2.6$, $1 \mathrm{H}, \mathrm{CH}), 7.18-7.65$ (m, 8H, Ar-H), $7.66(\mathrm{~d}, J=2.6,1 \mathrm{H}, \mathrm{CH})$; ${ }^{13} \mathrm{C}-\mathrm{NMR}\left(\mathrm{CDCl}_{3}\right) \delta 48.67,62.45,67.60,92.92,117.51,126.74$, 127.87, 128.06, 128.25, 128.79, 129.31, 130.42, 132.92, 133.79, $134.95,136.32,139.58,156.92,163.83, \mathrm{MS} m / z$ 414.0( $\left.\mathrm{M}^{+}\right)$, 176.0; Anal. calcd for $\mathrm{C}_{19} \mathrm{H}_{16} \mathrm{BrN}_{3} \mathrm{O}_{3}$ : C 55.09, H 3.89, N 10.14, found $\mathrm{C} 55.05, \mathrm{H} 3.91, \mathrm{~N} 10.12$.

3-(2-((1-(4-(Trifluoromethoxy)phenyl)-2H-pyrazol-3-yloxy) methyl)phenyl)oxazolidin-2-one (9i): A white solid, yield 87.5\%, mp $123-125^{\circ} \mathrm{C},{ }^{1} \mathrm{H}-\mathrm{NMR}\left(\mathrm{CDCl}_{3}\right) \delta 4.02\left(\mathrm{t}, J=7.9,2 \mathrm{H}, \mathrm{CH}_{2}\right)$, $4.47\left(\mathrm{t}, J=7.9,2 \mathrm{H}, \mathrm{CH}_{2}\right), 5.36\left(\mathrm{~s}, 2 \mathrm{H}, \mathrm{CH}_{2}\right), 5.93(\mathrm{~d}, J=2.6$, $1 \mathrm{H}, \mathrm{CH}), 7.23-7.64(\mathrm{~m}, 8 \mathrm{H}, \mathrm{Ar}-\mathrm{H}), 7.71(\mathrm{~d}, J=2.6,1 \mathrm{H}, \mathrm{CH})$; ${ }^{13} \mathrm{C}-\mathrm{NMR}\left(\mathrm{CDCl}_{3}\right) \delta 48.77,62.50,67.83,94.49,118.89,122.11$, $126.45,126.78,126.99,127.95,128.39$, 129.52, 130.59, 131.39, 134.81, 136.35, 138.61, 146.44, 156.92, 16427; MS m/z 420.1 $\left(\mathrm{M}^{+}\right)$, 176.1; Anal. calcd for $\mathrm{C}_{20} \mathrm{H}_{16} \mathrm{~F}_{3} \mathrm{~N}_{3} \mathrm{O}_{4}$ : C 57.28, H 3.85, $\mathrm{N} 10.02$, found C 57.26, H 3.87, N 9.98.

3-(2-((1-o-Tolyl-2H-pyrazol-3-yloxy)methyl)phenyl)oxazolidin-2-one (9j): A white solid, yield $86.0 \%$; mp $98-101{ }^{\circ} \mathrm{C}$, ${ }^{1} \mathrm{H}-\mathrm{NMR}\left(\mathrm{CDCl}_{3}\right) \delta 2.25\left(\mathrm{~S}, 3 \mathrm{H}, \mathrm{CH}_{3}\right), 3.98\left(\mathrm{t}, J=7.9,2 \mathrm{H}, \mathrm{CH}_{2}\right)$, $4.41\left(\mathrm{t}, J=7.9,2 \mathrm{H}, \mathrm{CH}_{2}\right), 5.30\left(\mathrm{~s}, 2 \mathrm{H}, \mathrm{CH}_{2}\right), 5.86(\mathrm{~d}, J=2.5$, $1 \mathrm{H}, \mathrm{CH}), 7.21-7.39$ (m, 8H, Ar-H), 7.61 (dd, $J=2.5,1 \mathrm{H}, \mathrm{CH})$; ${ }^{13} \mathrm{C}-\mathrm{NMR}\left(\mathrm{CDCl}_{3}\right) \delta 18.15,48.80,62.40,67.53,91.98,125.60$, $126.45,126.67,127.76,128.17,129.19,130.29,131.27,131.82$, 133.29, 135.01, 136.21, 139.79, 156.88, 163.38. MS m/z 350.1 $(\mathrm{M}+1), 176.1$; Anal. calcd for $\mathrm{C}_{20} \mathrm{H}_{19} \mathrm{~N}_{3} \mathrm{O}_{3}: \mathrm{C} 68.75, \mathrm{H} 5.48, \mathrm{~N}$ 12.03, found C 68.72, H 5.51, N 11.99.

3-(2-((1-(4-Fluoro-3-(trifluoromethyl)phenyl)-2H-pyrazol3-yloxy)methyl)phenyl)oxazolidin-2-one (9k): A white solid,
Table 1. Structures and fungicidal activities in vitro of compounds 9a-9l

\begin{tabular}{|c|c|c|c|c|c|c|}
\hline \multirow[b]{2}{*}{ No. } & \multirow{2}{*}{$\frac{\text { Compounds }}{\mathrm{R}}$} & \multicolumn{5}{|c|}{ Inhibition of growth ${ }^{a}\left(10 \mathrm{mg} \mathrm{L}^{-1}\right)$} \\
\hline & & $\begin{array}{c}G . \\
\text { zeae }\end{array}$ & $\begin{array}{c}R . \\
\text { cerealis }\end{array}$ & $\begin{array}{c}S . \\
\text { Sclerotioru }\end{array}$ & $\begin{array}{c}B . \\
\text { cinerea }\end{array}$ & $\begin{array}{c}M . \\
\text { grisea }\end{array}$ \\
\hline 9a & $\mathrm{H}$ & - & - & - & - & - \\
\hline $9 b$ & $4-\mathrm{Cl}$ & - & - & - & - & + \\
\hline $9 c$ & $3-\mathrm{Cl}$ & - & - & - & - & + \\
\hline 9d & $4-\mathrm{Br}$ & - & - & - & - & - \\
\hline $9 e$ & $3-F$ & + & - & + & + & + \\
\hline 9f & $2-\mathrm{CH}_{2} \mathrm{CH}_{3}$ & - & - & - & - & - \\
\hline $9 \mathrm{~g}$ & $2-\mathrm{Cl}$ & - & + & - & - & + \\
\hline $9 \mathrm{~h}$ & $2-\mathrm{Br}$ & - & - & - & - & - \\
\hline $9 \mathbf{i}$ & $4-\mathrm{OCF}_{3}$ & - & - & - & - & + \\
\hline $9 \mathbf{j}$ & $2-\mathrm{CH}_{3}$ & - & - & - & - & - \\
\hline $9 \mathbf{k}$ & $3-\mathrm{CF}_{3}-4-\mathrm{F}$ & - & + & - & - & + \\
\hline 91 & $3-\mathrm{CH}_{3}$ & - & - & - & - & - \\
\hline $\begin{array}{l}\text { pyraclo } \\
\text { strobin }\end{array}$ & - & +++ & +++ & +++ & +++ & +++ \\
\hline
\end{tabular}

${ }^{a}$ Activity is expressed in four categories: $(-)<50 \%,(+) 51-70 \%,(++) 71-$ $90 \%,(+++)>90 \%$.

Table 2. Structures and fungicidal activities in vivo of compounds 9a-9l

\begin{tabular}{|c|c|c|c|c|}
\hline \multirow{2}{*}{ No. } & \multirow{2}{*}{$\frac{\text { Compounds }}{\mathrm{R}}$} & \multicolumn{3}{|c|}{ Inhibition of growth $^{a}\left(1 \mathrm{~g} \mathrm{~L}^{-1}\right)$} \\
\hline & & S. Sclerotioru & B. cinerea & M. grisea \\
\hline 9a & $\mathrm{H}$ & - & - & ++ \\
\hline $9 b$ & $4-\mathrm{Cl}$ & - & - & + \\
\hline $9 \mathrm{c}$ & $3-\mathrm{Cl}$ & - & - & + \\
\hline 9d & $4-\mathrm{Br}$ & - & - & - \\
\hline $9 e$ & $3-\mathrm{F}$ & - & ++ & + \\
\hline 9f & $2-\mathrm{CH}_{2} \mathrm{CH}_{3}$ & - & - & - \\
\hline $9 \mathrm{~g}$ & $2-\mathrm{Cl}$ & - & - & ++ \\
\hline $9 \mathrm{~h}$ & $2-\mathrm{Br}$ & - & - & - \\
\hline $9 \mathbf{i}$ & $4-\mathrm{OCF}_{3}$ & - & - & + \\
\hline $9 \mathbf{j}$ & $2-\mathrm{CH}_{3}$ & - & - & - \\
\hline 9k & $3-\mathrm{CF}_{3}-4-\mathrm{F}$ & - & - & - \\
\hline 91 & $3-\mathrm{CH}_{3}$ & - & - & - \\
\hline Pyraclostrobin & - & +++ & ++ & +++ \\
\hline Carbendazol & - & +++ & ++ & \\
\hline
\end{tabular}

${ }^{a}$ Activity is expressed in four categories: $(-)<50 \%,(+) 51-70 \%,(++) 71-$ $90 \%,(+++)>90 \%$.

yield $88.5 \%$, mp $116-119{ }^{\circ} \mathrm{C},{ }^{1} \mathrm{H}-\mathrm{NMR}\left(\mathrm{CDCl}_{3}\right) \delta 4.04(\mathrm{t}, J=$ $\left.7.9,2 \mathrm{H}, \mathrm{CH}_{2}\right), 4.48\left(\mathrm{t}, J=7.9,2 \mathrm{H}, \mathrm{CH}_{2}\right), 5.36\left(\mathrm{~s}, 2 \mathrm{H}, \mathrm{CH}_{2}\right), 5.96$ $(\mathrm{d}, J=2.6,1 \mathrm{H}, \mathrm{CH}), 7.23-7.77(\mathrm{~m}, 8 \mathrm{H}, \mathrm{Ar}-\mathrm{H}), 7.80$ (dd, $J=$ 2.6, 1H, CH); ${ }^{13} \mathrm{C}-\mathrm{NMR}\left(\mathrm{CDCl}_{3}\right) \delta 48.72,62.49,67.88,95.02$, 116.45, 117.81, 118.11, 122.59, 122.71, 123.98, 126.68, 128.01, $128.37,129.52,130.46,134.70,136.28,155.29,156.89,164.42$. MS $m / z(\%) 422.1\left(\mathrm{M}^{+}\right), 176.1$; Anal. calcd for $\mathrm{C}_{20} \mathrm{H}_{15} \mathrm{~F}_{4} \mathrm{~N}_{3} \mathrm{O}_{3}$ : C 57.01, H 3.59, N 9.97,found C 56.97, H 3.62, N 9.96.

3-(2-((1-m-Tolyl-2H-pyrazol-3-yloxy)methyl)phenyl)oxazolidin-2-one (9I): A white solid, yield $81.5 \%$, mp $97-98{ }^{\circ} \mathrm{C}$, ${ }^{1} \mathrm{H}-\mathrm{NMR}\left(\mathrm{CDCl}_{3}\right) \delta 2.39\left(\mathrm{~S}, 3 \mathrm{H}, \mathrm{CH}_{3}\right), 4.02\left(\mathrm{t}, J=7.9,2 \mathrm{H}, \mathrm{CH}_{2}\right)$, $4.45\left(\mathrm{t}, J=7.9,2 \mathrm{H}, \mathrm{CH}_{2}\right), 5.34\left(\mathrm{~s}, 2 \mathrm{H}, \mathrm{CH}_{2}\right), 5.89(\mathrm{~d}, J=2.5$, $1 \mathrm{H}, \mathrm{CH}), 7.01-7.64(\mathrm{~m}, 8 \mathrm{H}, \mathrm{Ar}-\mathrm{H}), 7.71(\mathrm{~d}, J=2.5,1 \mathrm{H}, \mathrm{CH})$; ${ }^{13} \mathrm{C}-\mathrm{NMR}\left(\mathrm{CDCl}_{3}\right) \delta 31.45,48.76,62.48,67.69,93.53,114.91$, 
$118.62,126.23,126.78,127.83,128.32,129.10,129.42,130.61$, 134.94, 136.35, 139.36, 139.99, 156.98, 163.89; MS m/z 350.1 $\left(\mathrm{M}^{+}\right)$, 176.1; Anal. calcd for $\mathrm{C}_{20} \mathrm{H}_{19} \mathrm{~N}_{3} \mathrm{O}_{3}$ : C 68.75, $\mathrm{H}$ 5.48, N 12.03, found $\mathrm{C} 68.72, \mathrm{H} 5.51, \mathrm{~N} 12.00$.

X-ray diffraction crystallography. A suitable single crystal of 9a was obtained by dissolving the compound in ethyl acetate and evaporating the solvent slowly at room temperature for about $10 \mathrm{~d}$. The diffraction data were collected on a Nonius CAD4 single crystal diffractometer equipped with a graphite-mono chromated MoK $\alpha$ radiation $(\lambda=0.71073 \AA)$ by using an $\omega / 2 \theta$ scan mode at $296 \mathrm{~K}$. The crystal structure were solved by the direct method and refined by the full-matrix least-squares procedure on $F^{2}$ using SHELXL-97 program. ${ }^{24}$ All non-hydrogen atoms were refined anisotropically, and the hydrogen atoms were introduced at calculated positions.

Biological assay. The preliminary fungistatic activity of compounds 9a-9l against fungi Gibberella zeae, Rhizoctonia cerealis, Sclerotinia sclerotioru, Botrytis cinerea and Magnaporthe grisea in vitro were measured in a concentration of 10 $\mathrm{mg} \mathrm{L}^{-1}$ according to the literature. ${ }^{25}$ The in vivo preliminary fungistatic activities of compounds 9a-9l against Sclerotinia sclerotioru, Botrytis cinerea and Magnaporthe grisea were tested in a concentration of $1 \mathrm{~g} \mathrm{~L}^{-1}$ according to the literature. ${ }^{26}$ The results of in vitro and in vivo fungistatic activity were indicated in Table 1 and Table 2, respectively, compared with the activity of the commercial fungicide pyraclostrobin or carbendazol.

\section{Results and Discussion}

Synthesis and characterization. The synthetic route for the target compounds 9a-9l was outlined in Scheme 1. The intermediate 4a-4l were obtained through three steps. Substituted aniline (1a-11) via diazotization and reduction reaction got substituted phenyl hydrazine hydrochloride (2a-2l), and then $\mathbf{2 a -}$ 21 reacted with ethyl acrylate in the presence of $\mathrm{NaOCH}_{3}$ to give 1-substituted pyrazolidin-3-one (3a-3l). Then 3a-3l was oxidized by $\mathrm{O}_{2}$ in the presence of $\mathrm{FeCl}_{3} \cdot 6 \mathrm{H}_{2} \mathrm{O}$ as catalyst to give the intermediate 1-substituted phenyl-2H-pyrazolin-3-one (4a-4l).

Compounds 1-substituted pyrazolidin-3-one were synthesized according to the method reported in literature, ${ }^{19}$ but got low- er yields. When we sought to conduct this reaction at a temperature of $30{ }^{\circ} \mathrm{C}$, the desired product $\mathbf{3 b}$ was obtained with a yield of $25.6 \%$. When the reaction temperature was further increased to $45{ }^{\circ} \mathrm{C}$, the reaction yield increased to $78.8 \%$ by varying the reaction time. Furthermore, under the anhydrous reaction condition, the yield of pyrazolidin-3-one $\mathbf{3 b}$ is higher than the hydrous reaction. The detailed yields under different conditions as are listed in Table 3.

3-o-Tolyloxazolidin-2-one (7) was reacted with N-bromosuccinimide in the presence of azobisisobutyronitrile as an initiator in the $\mathrm{CCl}_{4}$ solution at reflux temperature without light to give 3-(2-bromomethylphenyl) oxazolidin-2-one (8). This reaction was a radical reaction, which yield was directly affected by reaction conditions. When the reaction temperature was below the lowest initiating temperature of AIBN, the reaction couldn't start. When the reaction temperature was much higher exceed than the initiating temperature of initiator, the reaction was difficult to control and got low yield. Under the reflux temperature of carbon tetrachloride $\left(74{ }^{\circ} \mathrm{C}-76{ }^{\circ} \mathrm{C}\right),{ }^{5}$ the reaction can be induced well by AIBN. In addition, the dark condition could conducive for this reaction, which yield could increase to $60 \%$.

The target compounds 9a-9l were obtained by the reaction of 3-(2-bromomethylphenyl)oxazolidin-2-one (8) with 1-sub-

Table 3. Optimization of reaction conditions for preparation of compound 3b

\begin{tabular}{cccccc}
\hline Entry & Product & Condition & Time $(\mathrm{h})$ & Temp $\left({ }^{\circ} \mathrm{C}\right)$ & Yield $(\%)$ \\
\hline 1 & $\mathbf{3 b}$ & anhydrous & 5 & 30 & 10.1 \\
2 & $\mathbf{3 b}$ & anhydrous & 10 & 30 & 18.7 \\
3 & $\mathbf{3 b}$ & anhydrous & 15 & 30 & 25.6 \\
4 & $\mathbf{3 b}$ & anhydrous & 5 & 45 & 45.4 \\
5 & $\mathbf{3 b}$ & anhydrous & 10 & 45 & 70.1 \\
6 & $\mathbf{3 b}$ & anhydrous & 15 & 45 & 78.8 \\
7 & $\mathbf{3 b}$ & anhydrous & 5 & 55 & 25.1 \\
8 & $\mathbf{3 b}$ & anhydrous & 10 & 55 & 28.8 \\
9 & $\mathbf{3 b}$ & anhydrous & 15 & 55 & 30.7 \\
10 & $\mathbf{3 b}$ & hydrous & 5 & 45 & 5.1 \\
11 & $\mathbf{3 b}$ & hydrous & 10 & 45 & 7.1 \\
12 & $\mathbf{3 b}$ & hydrous & 15 & 45 & 7.5 \\
\hline
\end{tabular}

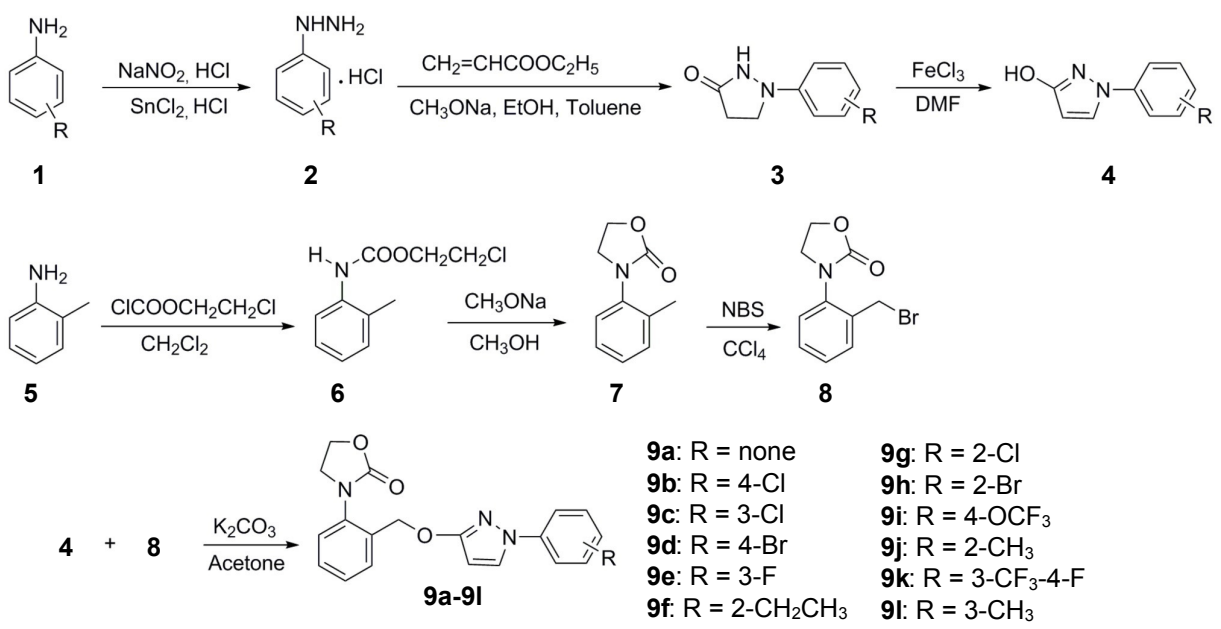

Scheme 1. General synthetic route for the target compounds 9a-91 


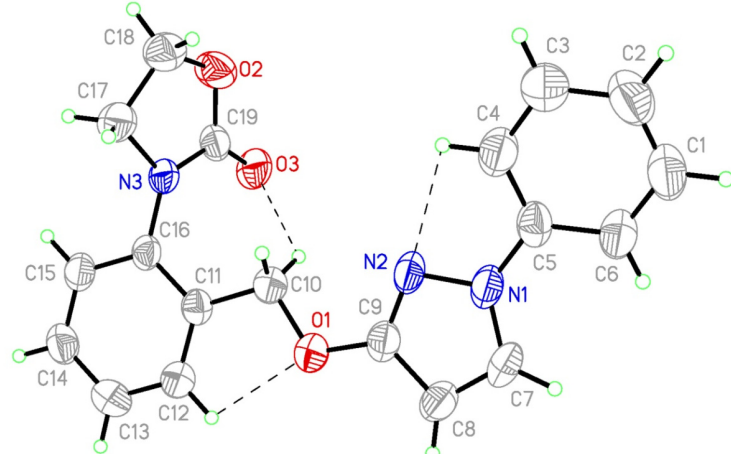

Figure 2. A molecule structure of 9a. Thin dashed lines represent intramolecular hydrogen bonds.

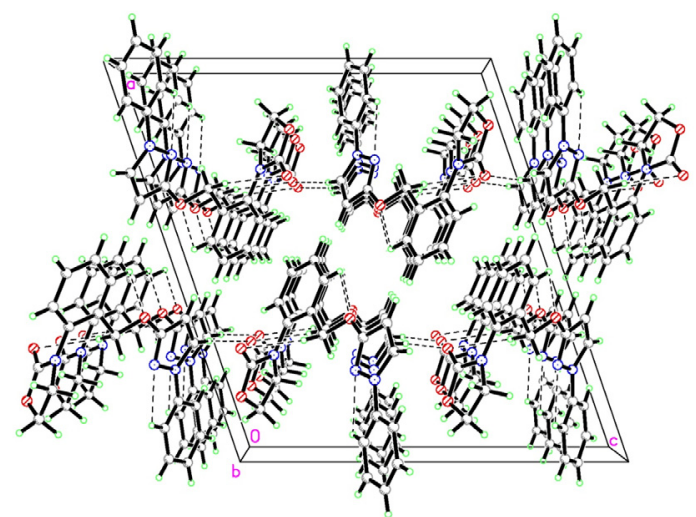

Figure 3. A packing plot of 9a viewed along the crystallographic $b$-axis.

stituted phenyl-2H-pyrazolin-3-one derivatives (4a-4I) in the presence of $\mathrm{K}_{2} \mathrm{CO}_{3}$ in the solution of acetone at boiling temperature in good yields between $79.6 \%-89.0 \%$. The structures of the target compounds 9a-9l were confirmed by elemental analyses, ${ }^{1} \mathrm{H}-\mathrm{NMR},{ }^{13} \mathrm{C}-\mathrm{NMR}$ and MS spectra. Furthermore, the structure of $9 \mathrm{a}$ was revealed by X-ray crystallography to understand the structure-fungicidal activity relationships.

Crystal structure. The crystal structure of $9 \mathrm{a}$ was determined by single-crystal X-ray diffraction, which belongs to monoclinic system, $P 2_{l} / C$ space group with unit cell parameters: $a=17.788$ (4) $\AA, b=6.0100$ (12) $\AA, c=16.222$ (3) $\AA$, and $\beta=108.87$ (3) . The detailed crystallographic data for $9 \mathbf{a}$ are collected in Table 4, and the hydrogen bonds were shown in Table 5.

The single crystal structure and packing diagram of $\mathbf{9 a}$ are shown in Figures 2 and Figures 3. The 2H-pyrazol ring and oxazolidin-2-one ring are twisted $6.04^{\circ}$ and $65.51^{\circ}$ from the plane of the bridge benzene ring ( $\mathrm{C} 11$ to $\mathrm{C} 16)$, respectively, whereas the side chain benzene group $(\mathrm{C} 1$ to $\mathrm{C} 6)$ are oriented at a dihedral angles of $22.39^{\circ}$ from the plane of the bridge benzene ring. The intramolecular $\mathrm{C}-\mathrm{H} \cdots \mathrm{N}$ and $\mathrm{C}-\mathrm{H} \cdots \mathrm{O}$ hydrogen bonds (Table 4) result in the formation of three non-planar pseudo rings $\mathrm{A}(\mathrm{C} 4 / \mathrm{C} 5 / \mathrm{N} 1 / \mathrm{N} 2 / \mathrm{H} 4 \mathrm{~A}), \mathrm{B}(\mathrm{O} 1 / \mathrm{C} 10 / \mathrm{C} 11 / \mathrm{C} 12 / \mathrm{H} 12 \mathrm{~A})$, and $\mathrm{C}(\mathrm{O} 3 / \mathrm{C} 19 / \mathrm{N} 3 / \mathrm{C} 16 / \mathrm{C} 11 / \mathrm{C} 10 / \mathrm{H} 10 \mathrm{~A})$. In the molecular packing of $9 \mathrm{a}$, intermolecular $\mathrm{C}-\mathrm{H} \cdots \mathrm{O}$ hydrogen bonds link the molecules stacked along the $b$ axis. The single crystal structure of the compound can provide a basis for elucidating the effect on
Table 4. Crystal and structure refinement data of compound 9a

\begin{tabular}{|c|c|}
\hline Compound & $9 a$ \\
\hline Chemical formula & $\mathrm{C}_{19} \mathrm{H}_{17} \mathrm{~N}_{3} \mathrm{O}_{3}$ \\
\hline Formula weight & 335.36 \\
\hline Crystal system & Monoclinic \\
\hline Space group & $P 2_{1} / C$ \\
\hline$a(\AA)$ & $17.788(4)$ \\
\hline$b(\AA)$ & $6.0100(12)$ \\
\hline$c(\AA)$ & $16.222(3)$ \\
\hline$\beta\left(^{\circ}\right)$ & $108.87(3)$ \\
\hline$V\left(\AA^{3}\right), Z$ & $1641.0(6) / 4$ \\
\hline$D_{\text {calc }}\left(\mathrm{g} \mathrm{cm}^{-3}\right)$ & 1.357 \\
\hline$\mu\left(\mathrm{mm}^{-1}\right)$ & 0.09 \\
\hline$F\left(\begin{array}{lll}0 & 0 & 0\end{array}\right)$ & 704 \\
\hline$\theta$ range $\left(^{\circ}\right)$ & $9-13$ \\
\hline Index range & $\begin{array}{c}-20 \leq h \leq 0 \\
0 \leq k \leq 7 \\
-18 \leq l \leq 19\end{array}$ \\
\hline Reflections collected & 3074 \\
\hline Unique reflections $\left(R_{\text {int }}\right)$ & $2972(0.029)$ \\
\hline Refinement mothod on $F^{2}$ & Full-matrix least-squares \\
\hline GOF on $F^{2}$ & 1.000 \\
\hline$R_{I}[I>2 \sigma(I)]$ & 0.1839 \\
\hline$w R_{2}[I>2 \sigma(I)]$ & 0.1503 \\
\hline$R_{l}$ (all data) & 0.1104 \\
\hline$w R_{2}$ (all data) & 0.0588 \\
\hline Residual (e $\left.\AA^{-3}\right)$ & 0.210 and -0.178 \\
\hline
\end{tabular}

Table 5. Hydrogen-bond geometry of compound $9 \mathbf{a}\left(\AA,^{\circ}\right)$

\begin{tabular}{ccccc}
\hline $\mathrm{D}-\mathrm{H} \cdots \mathrm{A}$ & $\mathrm{D}-\mathrm{H}$ & $\mathrm{H} \cdots \mathrm{A}$ & $\mathrm{D} \cdots \mathrm{A}$ & $\mathrm{D}-\mathrm{H} \cdots \mathrm{A}$ \\
\hline $\mathrm{C} 4-\mathrm{H} 4 \mathrm{~A} \cdots \mathrm{N} 2$ & 0.93 & 2.45 & $2.780(5)$ & 101 \\
$\mathrm{C} 7-\mathrm{H} 7 \mathrm{~A} \cdots \mathrm{O} 3^{\mathrm{i}}$ & 0.93 & 2.51 & $3.439(4)$ & 174 \\
$\mathrm{C} 10-\mathrm{H} 10 \mathrm{~A} \cdots \mathrm{O} 3$ & 0.97 & 2.55 & $3.255(3)$ & 129 \\
$\mathrm{C} 12-\mathrm{H} 12 \mathrm{~A} \cdots \mathrm{O} 1$ & 0.93 & 2.34 & $2.708(4)$ & 103
\end{tabular}

Symmetry codes: (i) $\mathrm{x},-\mathrm{y}+3 / 2, \mathrm{z}^{-1 / 2}$.

their biological activities.

Fungicidal activities. The in vitro fungicidal results of all these compounds against G. zeae, R. cerealis, S. Sclerotioru, B. cinerea and M. grisea were listed in Table 1. As compared with pyraclostrobin, most of compounds showed weak fungicidal activity against $G$. zeae, R. cerealis, S. Sclerotioru and B. cinerea at the concentration of $10 \mathrm{mg} \mathrm{L}^{-1}$, but half of the compounds $(9 \mathrm{~b}, 9 \mathrm{c}, 9 \mathrm{e}, 9 \mathrm{~g}, 9 \mathrm{i}, 9 \mathrm{k})$ showed moderate fungicidal activities against $M$. grisea, the common point of these structures contained electron withdrawing groups. The results showed that molecular structure contained electron withdrawing groups would enhanced the fungicidal activity against $M$. grisea. It is worth mentioning that the compound $\mathbf{9 e}$ containing 3-F group showed a broader antifungal activity spectrum against $G$. zeae, $S$. Sclerotioru, B. cinerea and M. grisea in vitro than other compounds.

The fungicidal results of all these compounds in vivo against 
S. Sclerotioru, B. cinerea and M. grisea were compared with two commercial fungicides pyraclostrobin and carbendazol. As shown in Table 2, at the concentration of $1 \mathrm{~g} \mathrm{~L}^{-1}$, none of the compounds showed good fungicidal activity against S. Sclerotioru. Compound $9 \mathrm{e}$ showed good fungicidal activities against $B$. cinerea, the inhibition rate is within $71-90 \%$. The fungicidal activities against $M$. grisea of the compounds 9a-9l are influenced by the nature of the $\mathrm{R}$ group in phenyl. When $\mathrm{R}$ group was $\mathrm{H}$ or 2-Cl, compounds 9a and 9g showed higher fungicidal activity, the inhibition rate are within $71-90 \%$. When $\mathrm{R}$ group were 4-Cl, 3-Cl, 3-F, 4-OCF 3 , compounds $9 b, 9 c, 9 e$ and $9 \mathbf{i}$ showed moderate fungicidal activity, and the inhibition rate are within $51-70 \%$. It is worth to attention that 3-F substituent analogue (9e) showed good fungicidal activities, and the in vivo bioassay results are consistent with the in vitro bioassay results. The reason was speculated that $F$ was a electron withdrawing group which could balanced the HLB value of molecule and increased the systemic of molecule within plant. By contrast, compound $9 \mathbf{k}$ represented different results, which showed weak fungicidal activities against $S$. Sclerotioru, B. cinerea and $M$. grisea, the inhibition rate is below $50 \%$. It was supposed that $3-\mathrm{CF}_{3}-4-\mathrm{F}$ substituents in 9k were increased the HLB value of molecule and cut down the systemic of molecule. Another reason would be the steric effect of $\mathrm{CF}_{3}$ group changed the spatial configuration of molecule. Aimed to enhance the fungicidal activities, analyzed the fungicidal results, a rule has been revealed that balance the HLB value of molecule by modifying the R group in phenyl from a hydrogen atom to electron-withdrawing group and enhance the systemic of molecule by changing the spatial configuration of molecule as little as possible.

On the other hand, fungicidal results and the crystal structure of $9 \mathbf{a}$ (Figure 2 ) showed that the carbonyl oxygen moiety of the toxophore with other atoms formed a non-planar pseudo ring $\mathrm{C}(\mathrm{O} 3 / \mathrm{C} 19 / \mathrm{N} 3 / \mathrm{C} 16 / \mathrm{C} 11 / \mathrm{C} 10 / \mathrm{H} 10 \mathrm{~A})$. According to published crystal structures of the cytochrome bc1 enzyme complex and co-crystallization of the bovine heart enzyme with different Qo inhibitors, ${ }^{13}$ the carbonyl oxygen moiety in oxazolidin-2one ring couldn't bind to the amide group of glutamine with a hydrogen bond, so the fungicidal activity is mediocre. The structure-fungicidal activity relationships showed a rule of key point on the design of new compounds that avoid form an intramolecular pseudo ring containing active fungicidal group in molecular structure.

\section{Conclusion}

In conclusion, we have demonstrated the molecular design, synthesis, and fungicidal activities of a series of new type oxazolidin-2-one derivatives containing $\mathrm{N}$-substituted phenyl $2 \mathrm{H}$ pyrazol ring, and the crystal structure of the compound 3-(2((1-phenyl-2H-pyrazol-3-yloxy)methyl)phenyl)oxazolidin-2one (9a) was determined by single crystal X-ray diffraction analysis. The preliminary bioassay showed that some of the $\mathrm{N}$-substituted phenyl $2 \mathrm{H}$-pyrazol oxazolidin-2-one derivatives(9a-91) exhibited potential in vivo fungicidal activities against $M$. gri- sea at the dosage of $1 \mathrm{~g} \mathrm{~L}^{-1}$. The relationship between fungicidal activities and core structure showed that active fungicidal group formed intramolecular hydrogen bond in molecular structure. Expecting to find some new type strobilurin fungicides with high activities and low toxicities, further structural optimization and fungicidal activities about the oxazolidinone analogues are well under way.

\section{References}

1. Takase, A.; Kai, H.; Nishida, K.; Masui, M. EP 5359281993.

2. Heinemann, U.; Gayer, H.; Gerdes, P.; Krueger, B. W.; Gallenkamp, B.; Stelzer, U.; Marhold, A.; Tiemann, R.; Stefan, D.; Gerd, H.; Stenzel, K. DE 196020951997.

3. Wenderoth, B.; Rentzea, C.; Ammermann, E.; Pommer, E. H.; Steglich, W.; Anke, T. DE 36239211988.

4. Mueller, B.; Koenig, H.; Kirstgen, R.; Oberdorf, K.; Roehl, F.; Goetz, N.; Sauter, H.; Lorenz, G.; Ammermann, E. DE 4423612 1996.

5. Li, Y.; Zhang, H.-Q.; Liu, J.; Yang, X.-P.; Liu, Z.-J. J. Agric. Food Chem. 2006, 54, 3636 .

6. Zhao, P.-L.; Liu, G.-L.; Huang, W.; Wang, Y.-Z.; Yang, G.-F. J. Agric. Food Chem. 2007, 55, 5697.

7. Liu, A.-P.; Wang, X.-G.; Ou, X.-M.; Huang, Z.-M.; Chen, C.; Liu, S.-D.; Huang, L.; Liu, X.-P.; Zhang, C.-L.; Zheng, Y.-Q.; Ren, Y.-G.; He, L.; Yao, J.-R. J. Agric. Food Chem. 2008, 56, 6562.

8. Tu, S.; Xu, L. H.; Ye, L.-L.; Wang, X.; Sha, Y.; Xiao, Z.-Y. J. Agric. Food Chem. 2008, 56, 5247.

9. Hwang, I.-C.; Kim, J.-K.; Kim, H.-H.; Kyung, S.-H. Bull. Korean Chem. Soc. 2009, 30, 1475.

10. Stefan, H.; Kai, S.; Harald, K.; Uwe, C. Plant Physiol. 2002, 130, 120.

11. Josep, V. M.; Celia, S. P.; Consuelo, A.; Antonio, A.; Antonio, A. F. J. Agric. Food Chem. 2008, 56, 7682.

12. Zhang, Z.-L.; Huang, L.; Shulmeister, V. M.; Chi, Y.; Kim, K. K.; Hung, L. W.; Croftsk, A. R.; Berry, E. A.; Kim, S. H. Science 1998, 281,64

13. Ulrich, G.; Helge, S.; Alison, C.; Alan, M. Pest Manag. Science 2002, 58,859 .

14. Zappia, G.; Menendez, P.; Delle, M. G.; Misiti, D.; Nevola, L.; Botta, B. Mini-Rev. Med. Chem. 2007, 7, 389.

15. Zappia, G.; Cancelliere, G.; Gacs, B. E.; Delle, M. G.; Misiti, D.; Nevola, L.; Botta, B. Curr. Org. Syn. 2007, 4, 238.

16. Renslo, A. R.; Luehr, G. W.; Gordeev, M. F. Bioorg. Med. Chem. 2006, 14, 4227.

17. Mukhtar, T. A.; Wright, G. D. Chem. Rev. 2002, 105, 529.

18. Michael, E.; Augustyniak, E. D.; Cochran, J. L. E.; Fang, X. Q.; David, S. G.; David, R. J. J. Med. Chem. 2007, 50, 6367.

19. Koenig, H.; Goetz, N.; Klein, U.; Eller, K. WO 9703969, 1997.

20. Fancher, L. W.; Gless, R. D. Tetrahedron Lett. 1988, 29, 5095.

21. Morita, Y.; Ishigaki, T.; Kawamura, K.; Iseki, K. Synthesis 2007, $16,2517$.

22. Gurdere, M. B.; Budak, Y.; Ceylan, M. Asian J. Chem. 2008, 20, 1425.

23. Martins, A. M.; Rory, N. G. S.; Jose, N. R. J.; Antonio, E. J.; Alicia G. B.; Antonio, R. M. F.; Vicente, J. A. ChemMedChem. 2009, 4, 78.

24. Sheldrick, G. M. SHELXL-97, Program for the Refinement of Crystal Structures, University of Göttingen, 1997.

25. Jarosław, W.; Ewa, K.; Janusz, J. S.; Zbigniew, J. B. Pest Manag. Sci. 2001, 57, 625.

26. Huang, W.; Zhao, P.-L.; Liu, G.-L.; Chen, Q.; Liu, Z.-M.; Yang, G.-f. J. Agric. Food Chem. 2007, 55, 3004. 